DESCRIPTION OF THE LAWRENCE LIVERMORE NATIONAL LABORATORY SYNTHETIC GAMMA RAY SOURCE

S. John Luke

April 4, 2011 
This document was prepared as an account of work sponsored by an agency of the United States government. Neither the United States government nor Lawrence Livermore National Security, LLC, nor any of their employees makes any warranty, expressed or implied, or assumes any legal liability or responsibility for the accuracy, completeness, or usefulness of any information, apparatus, product, or process disclosed, or represents that its use would not infringe privately owned rights. Reference herein to any specific commercial product, process, or service by trade name, trademark, manufacturer, or otherwise does not necessarily constitute or imply its endorsement, recommendation, or favoring by the United States government or Lawrence Livermore National Security, LLC. The views and opinions of authors expressed herein do not necessarily state or reflect those of the United States government or Lawrence Livermore National Security, LLC, and shall not be used for advertising or product endorsement purposes.

This work performed under the auspices of the U.S. Department of Energy by Lawrence Livermore National Laboratory under Contract DE-AC52-07NA27344. 


\title{
DESCRIPTION OF THE LAWRENCE LIVERMORE NATIONAL LABORATORY SYNTHETIC GAMMA RAY SOURCE
}

\author{
John Baker, Greg White, Bert Pohl and $\underline{\text { S. John Luke }}{ }^{1}$, LLNL
}

\begin{abstract}
The Lawrence Livermore National Laboratory synthetic gamma ray source is a portable, stand-alone system that generates signals simulating with high fidelity the signals that would be output from a high-purity germanium (HPGe) detector in response to gamma rays from a user-defined mixture of special nuclear materials. This document describes the basic system concept, lays out the methodology that defines the generation of signals, and explains the Graphical User Interface for the operation of the Synthetic Gamma Ray source. The appendix to this paper offers a procedure to operate the source.
\end{abstract}

\section{CONTENTS}

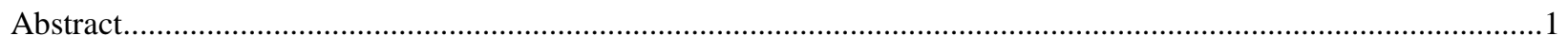

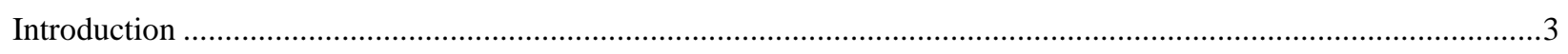

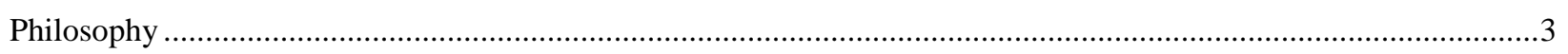

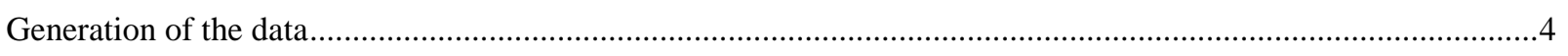

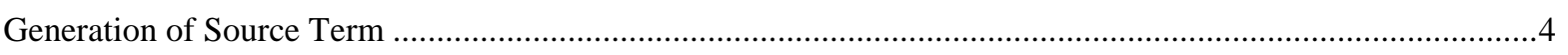

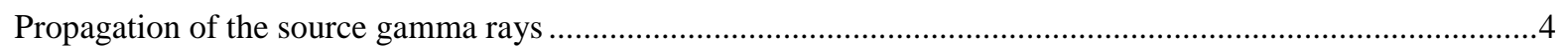

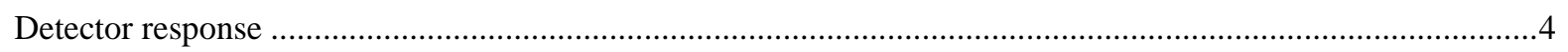

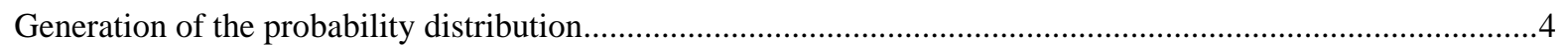

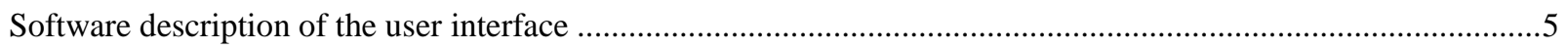

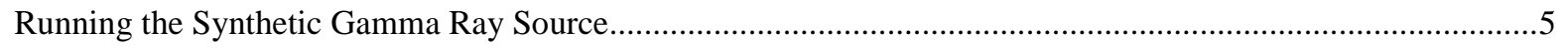

The Gamma Pulser start sequence .................................................................................................................

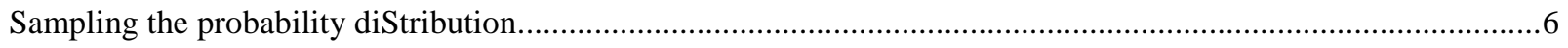

Spectral pulse generation algorithm including pileup......................................................................................6

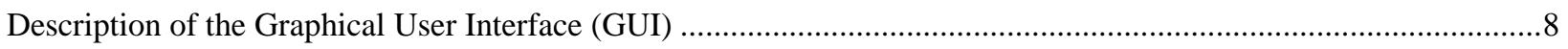

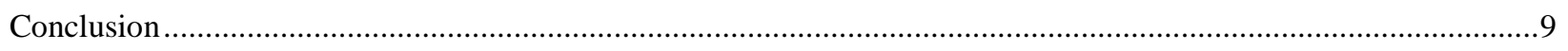

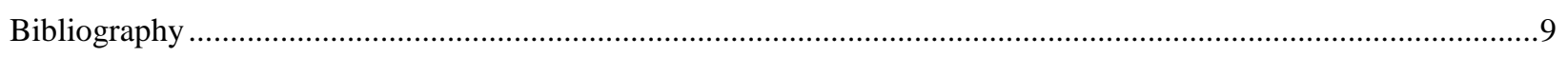

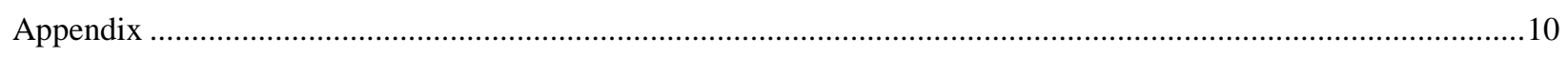

Synthetic Gamma Ray Source Set-up........................................................................................................

\footnotetext{
${ }^{1}$ Any questions about the synthetic gamma ray source should be directed to the author at luke2@llnl.gov.
} 
Setup Using Canberra Inspector and Genie Gamma Acquisition \& Analysis software ........................................10

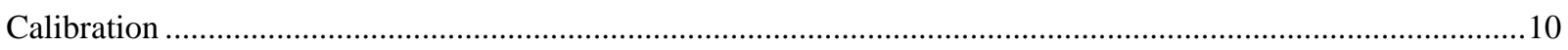

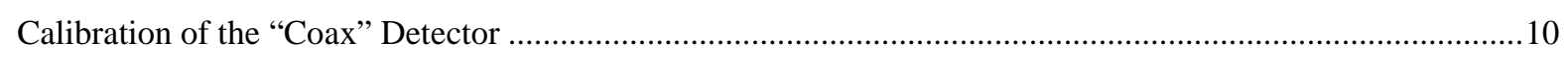

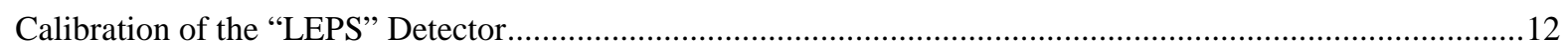




\section{INTRODUCTION}

The measurement of the gamma ray emission from various special nuclear materials (SNM) is necessary for the development of new analysis algorithms and for implementation of new hardware in existing measurement systems. Radiation measurements are also required for training new users of radiation measurement systems. A synthetic gamma ray source capable of accurately simulating detector response from actual nuclear material mixtures of interest offers a number of practical advantages, it allows significant algorithm-development, hardwareimplementation, and training activities to be carried out without the cost, hazards, and security requirements associated with handling actual special nuclear materials. It provides the flexibility to simulate an arbitrarily wide range of SNM mixtures. In addition, a synthetic gamma ray source could also be used for the authentication of the hardware and software of a measurement system in a controlled setting without the presence of any radiation sources. This will lead to more effective use of resources. The synthetic gamma ray source is intended to be a stand alone, portable unit.

\section{PHILOSOPHY}

This implementation of the synthetic gamma ray source has four major components:

1. Generation of the data

2. Software for the user interface

3. The field-programmable gate array (FPGA) hardware

4. The software running in the FPGA hardware

The overall structure of how the Synthetic Gamma Ray Source works is shown in Figure 1. The generation of the data occurs in the red box shown in the diagram in Figure 1. The interface software also generates a random number seed and does the sampling from the probability distribution. The randomly selected number is sent to the FPGA which outputs a random tail-pulse that has the correct characteristics to be input to any Multichannel Analyzer.

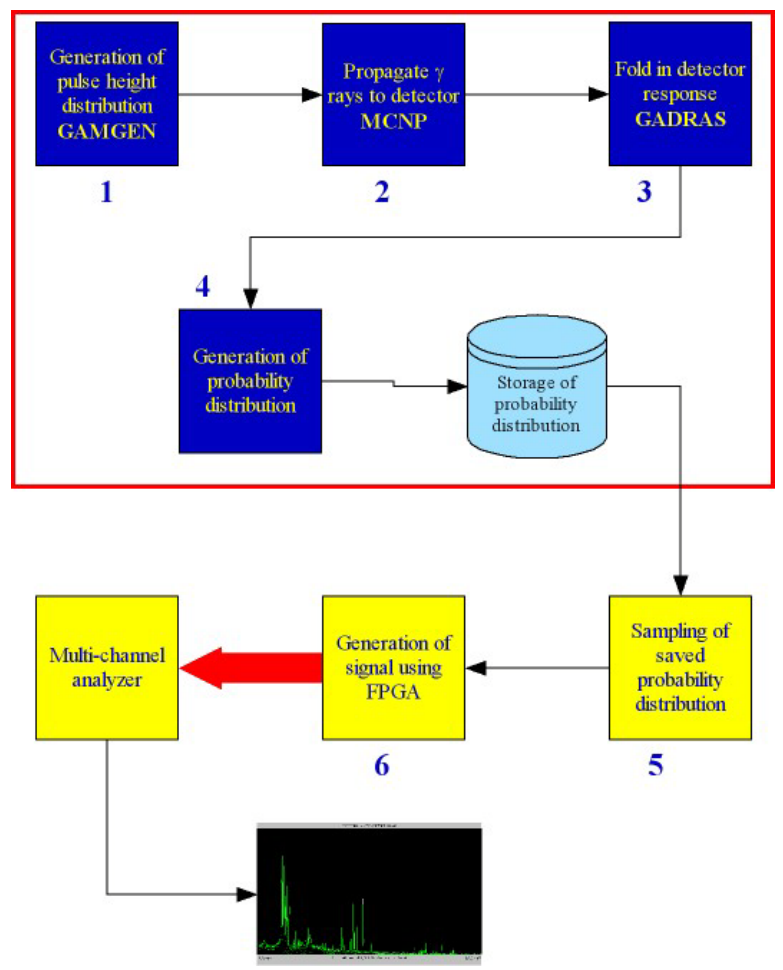

FIGURE 1: OVER STRUCTURE OF THE LOGIC OF THE SYNTHETIC GAMMA RAY SOURCE 


\section{GENERATION OF THE DATA}

As indicated above, the generation of the data for use in the Synthetic Gamma Ray Source is accomplished in four steps shown in the red box in Figure 1. These four steps are:

1. Generation of the gamma-ray energy distribution of the chosen source using GAMGEM.

2. Propagation of the source gamma rays through the appropriate shielding to the detector using MCNP.

3. Folding in the response of a detector using GADRAS.

4. Generation of a normalized probability distribution.

\section{Generation of SOURCE TERM}

The first step in the process of the Synthetic Gamma Ray Source is to generate the original source term. This is accomplished by using the code GAMGEM developed by Gosnell (Gosnell 1990). This code allows for the mixture of all the isotopes that would be present in a source at a certain time. For example, in generating the source term for a plutonium item all the isotopes are included $\left({ }^{236} \mathrm{Pu},{ }^{238} \mathrm{Pu},{ }^{241} \mathrm{Pu}\right.$, and $\left.{ }^{242} \mathrm{Pu}\right)$. The code computes the decay of the parent nuclides to a desired age and includes the gamma-ray contributions from all daughter nuclei. The resultant source term is used in the next step.

\section{PROPAGATION OF THE SOURCE GAMMA RAYS}

The source term obtained from the GAMGEM code is then used in MCNP (Breismeister 1991) to propagate the gamma rays from the source to the detector. The MNCP calculations assumed a one meter distance from the center of the source to the front face of the detector. Two types of shielding scenarios were calculated: a bare source and a source in a standard U.S. ALR8 container. The strength of the calibration sources were $10 \mathrm{nCi}$ with no correction for the life time of the source. The uranium and plutonium sources were assumed to be one-kilogram spheres. The energy of the gamma rays that impinge on the detector face are tallied and saved.

\section{DETECTOR RESPONSE}

The tallied results were used as input to the detector response code GADRAS (Mitchell 1992). GADRAS applies a physics-based response of the chosen detector to the incident gamma ray. The Synthetic Gamma Ray Source provides the signals for two different HPGe detectors: a 40-percent coax detector and a standard LEPS detector. Since the two detectors have different energy ranges that they are sensitive to, the energy ranges for the two detectors were limited. The coax detector had a maximum energy of three $\mathrm{MeV}$; while the LEPS detector had a maximum energy of $300 \mathrm{keV}$.

\section{GENERATION OF THE PROBABILITY DISTRIBUTION}

The output of the GADRAS code is a 8192-channel pulse height distribution representing the detector response to the user-selected source and shielding conditions. For a given channel $i$ in the pulse height distribution there would be $N_{i}$ counts in that channel. The total number of counts in a given MCNP calculation would be

$$
N_{T}=\sum_{i=1}^{8192} N_{i}
$$

The normalized probability $P_{i}$ for a count to be in channel $i$ would be

$$
P_{i}=\frac{N_{i}}{N_{T}} .
$$

$P$ is the probability distribution that is used in the sampling. 


\section{SOFTWARE DESCRIPTION OF THE USER INTERFACE}

\section{RunNING THE SyNTHETIC GAMMA RAY SOURCE}

An application named "Synthetic Gamma Ray Source" was developed as a simple user interface for the user to operate the electronic source. The software package consists of an executable named "Pulser.exe" and a library of sources. Running the executable opens a window as shown in Figure 2.

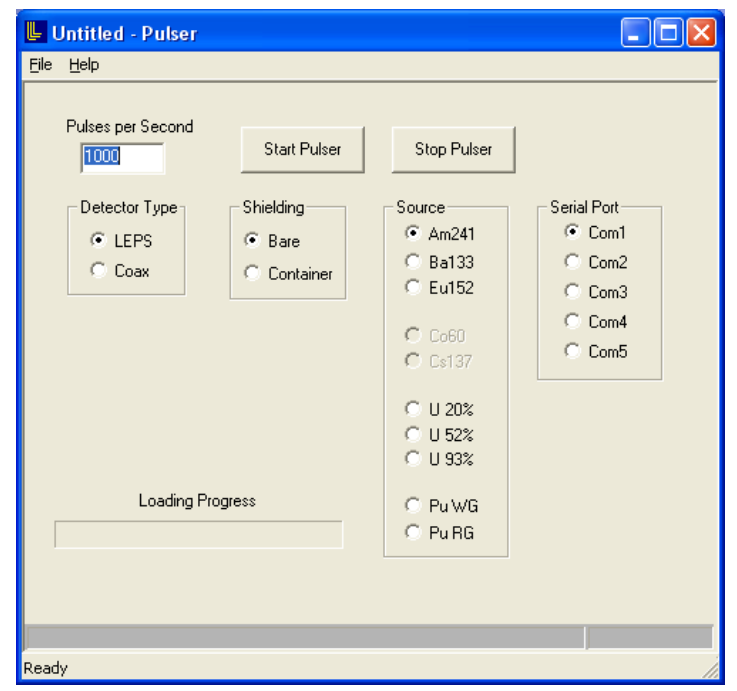

FIGURE 2: LLNL SYNTHETIC GAMMA RAY SOURCE INTERFACE.

\section{THE GAMMA PUlSER START SEQUENCE}

Each time the gamma pulser is started the following sequence of events is executed:

- Read the gamma input file corresponding to the gamma source selection

- Generate a new random number generator seed based on the time of day

- Compute the alias and cutoff values for the desired probability distribution (see discussion on this topic below)

- Send the following sequence of commands and data to the pulser:
o Reset Pulser
o Send Delay Time Array
o Send Amplitude Calibration Factor
o Send F Array
o Send IA Array
o Send First Seed
o Send Second Seed
o Start Pulser

The progress of each of these command sequences, some of which require several seconds to complete, is reported to the user as a series of text messages in a field at the bottom of the window. The pulser, following the transmission of each command, returns status to the program indicating either success or failure. All errors are reported to the user in a pop-up window. If an error occurs anywhere in the startup sequence, the pulser start is aborted. 


\section{SAMPLING THE PROBABILITY DISTRIBUTION}

In this implementation of the synthetic gamma ray source, the reproduction of the pulse height distribution is accomplished by means of a model calculation. Each point in the model pulse height distribution can be associated with a probability of population in relation to the spectrum as a whole. The goal then is to sample this probability distribution and produce a pulse corresponding to each sample. In order to generate the probability distribution, the pulse heights are normalized. As expected, the sum of the probabilities over the 8192 channels is unity.

Sampling the probability distribution quickly and efficiently is an art form that has generated a lot of interest in the past. The method developed by Alastair Walker (Walker 1977) was chosen because of its simplicity and ease of implementation (It was developed for applications running on computers with limited memory.) This method relies on an alias and cutoff method, which is a modification of rejection techniques. Let $\mathrm{x}$ be a random integer from the random number generator, uniformly distributed from 1 to $\mathrm{M}$, where $\mathrm{M}$ can be much larger than the number of channels, $\mathrm{N}$, over which we want to sample the pulse height distribution $\mathrm{P}(\mathrm{i})$. In the Walker method, the probability distribution is formed into a cutoff distribution $F(x)$ and an alias distribution $I A(x)$, where

$$
\begin{aligned}
& i=x \text { with probability } F(x) \text { and, } \\
& i=I A(x) \text { with probability } 1-F(x)
\end{aligned}
$$

Normalization in the algorithm ensures that the probability $P(i=j)=P(j)$, where $j=1$ to $N$, and $\Sigma P(j)=1$

Thus, for each $F(x)$, an alias $I A(x)$ is defined through pairing of low and high probabilities for each ' $x$ ' in the distribution. A random number between 1 and $N$ can now be generated to choose a channel in the distribution. A second random number is generated for comparison with the cutoff value. If the random number is less than or equal to the cutoff value, channel $x$ is increased. If the random number is greater than the cutoff value, the alias channel to $x$ is increased.

\section{SPECTRAL PULSE GENERATION ALGORITHM INCLUDING PILEUP}

An output of a single positive tail pulse is shown in Figure 3 below.

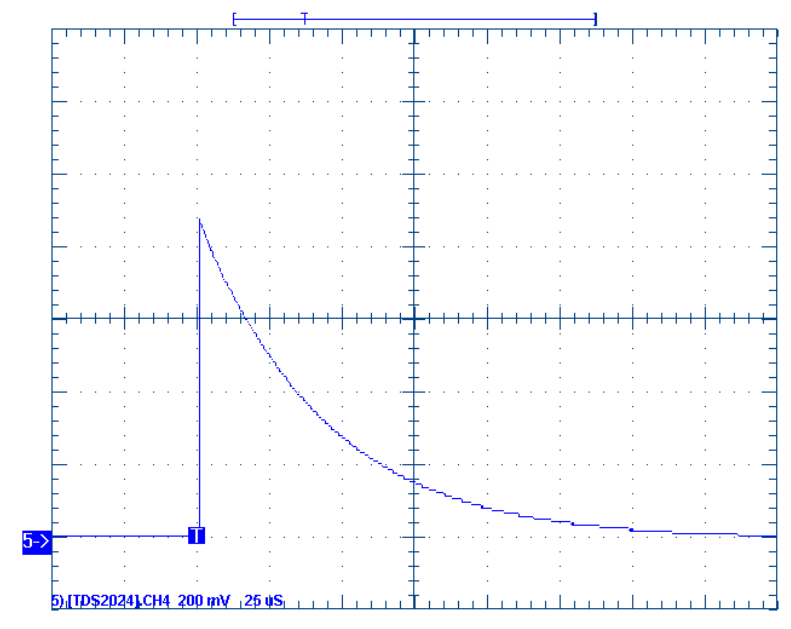

FIGURE 3: SINGLE POSITIVE TAIL PULSE

The PC code analyzes the spectrum according to Walker's algorithm and produces two integer data files, the $F$ array and the IA array, that are transmitted to the FPGA for further processing. The FPGA processes the $F$ array and the IA array to produce the tail pulse amplitudes that will regenerate the original spectrum. In Walker's algorithm, the $F$ 
array is a floating-point array. The floating-point array was converted to an array of 32-bit integers to allow integer processing in the FPGA. Since the input probability array was composed of 8192 points, the $F$ and $I A$ arrays have 8192 values as well.

The Synthetic Gamma Ray Source can be run in either a static timing distribution or a random timing distribution. Since the time between pulses is random, and the half-life of the isotopes is large as compared with the measurement time, a Poisson distribution is used for the timing between events. Knoll (Knoll 2000) presents a detailed discussion of the timing distributions. ${ }^{2}$

For the Poisson distribution, the time between tail-pulses is approximately:

$$
\Delta t=-\frac{1}{f} \cdot \ln U_{i}
$$

where $f$ is the average pulse frequency and $U_{i}$ are independent pseudo-random variables. The FPGA used in the Synthetic Gamma Ray source with a clock rate of $24 \mathrm{MHz}$, the number of clock cycles between tail-pulses is:

$$
N=\frac{24 \cdot 10^{6}}{f} \cdot \log _{e}\left(U_{i}\right)
$$

In order to set up the Poisson random timing of the tail-pulses, a third 128-point array for the tail-pulse Poisson timing is produced in the PC and transmitted to the FPGA. This timing array is an array of natural logarithms divided by the average pulse rate selected for the tail-pulses as shown above, modified slightly to allow for a minimum time interval of $3 \mu \mathrm{s}$, the minimum time interval that can be produced by the present implementation of the FPGA code.

A consequence of the random tail-pulse timing is the occurrence of pileup, the phenomenon whereby one or more tail-pulses have not decayed to zero before subsequent pulse(s) occur. A picture of this situation is shown in Figure 4.

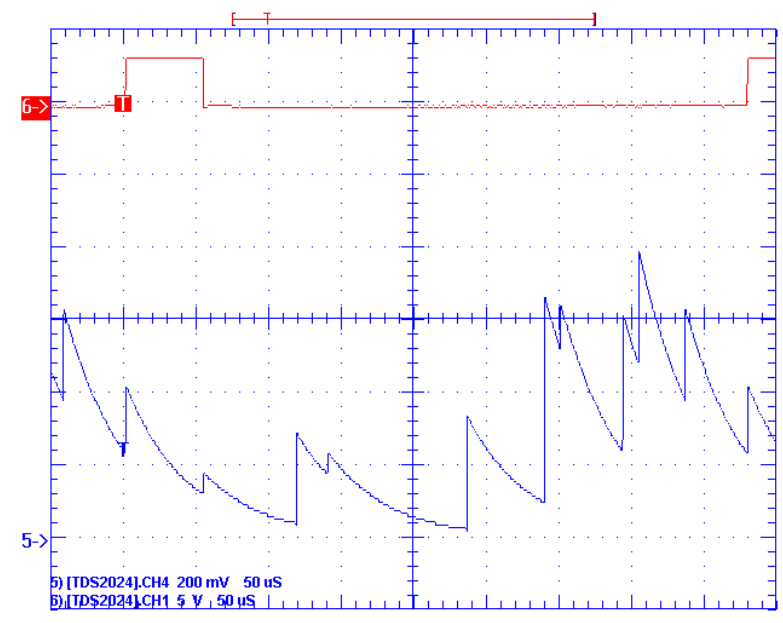

FIGURE 4: GENERATION OF POSITIVE TAIL PULSES - INCLUDING PILE-UP

Pileup is handled by simply adding the new tail-pulse amplitude to the old exponentially decaying tail-pulse, as in real-life, and then exponentially decaying the resulting signal. The Synthetic Gamma Ray Source shows the effect of

\footnotetext{
${ }^{2}$ See pages 97-8.
} 
pileup in the response of the detector. This is shown in the plot in Figure 5. In this figure the output of the Synthetic Gamma Ray Source is plotted for the ${ }^{137}$ Cs with a series of count rates. The plot in Figure 5 shows that the resolution of the signal worsens with the increased count rate in the detector as is expected.

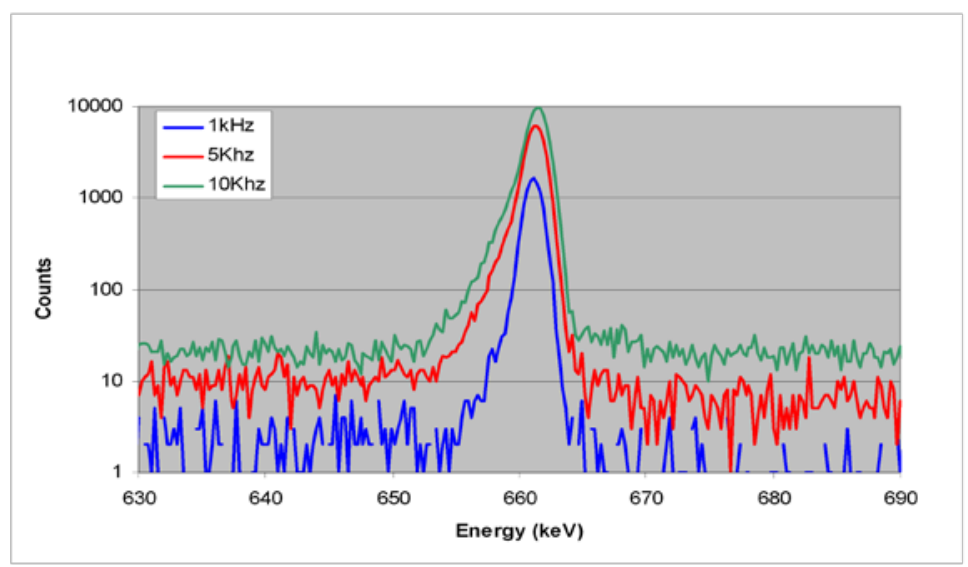

FIGURE 5: EFFECT OF COUNT RATE ON THE PEAK SHAPE FROM THE SYNTHETIC GAMMA RAY SOURCE

\section{DESCRIPTION OF THE GRAPHICAL USER INTERFACE (GUI)}

The GUI for the Synthetic Gamma Ray is shown in Figure 6. The GUI allows the user to select a number of variables.

1. The first selection (blue box) is the number of pulses per second; this allows the user to control the rate of the source.

2. The second user selected (red box) choice is the type of detector; the user can choose an LEPS or Coax detector. If the user selects an LEPS the ${ }^{60} \mathrm{Co}$ and ${ }^{137} \mathrm{Cs}$ cannot be selected. If the user chooses the Coax detector the ${ }^{241} \mathrm{Am},{ }^{133} \mathrm{Ba}$ and the ${ }^{152} \mathrm{Eu}$ cannot be selected. ${ }^{3}$

3. The third user (light blue) selected choice is the type of shielding - whether bare or in a container.

4. The fourth user (gold box) selected choice is the type of source.

5. The fifth user (purple box) selected choice is the serial port number on the PC.

${ }^{3}$ The ${ }^{60} \mathrm{Co}$ and ${ }^{137} \mathrm{Cs}$ sources cannot be used with the LEPS detector because the detector has no efficiency for observing these gamma rays. The choice not to include the ${ }^{241} \mathrm{Am},{ }^{133} \mathrm{Ba}$ and the ${ }^{152} \mathrm{Eu}$ sources for the Coax detector was just a choice by the developers. 


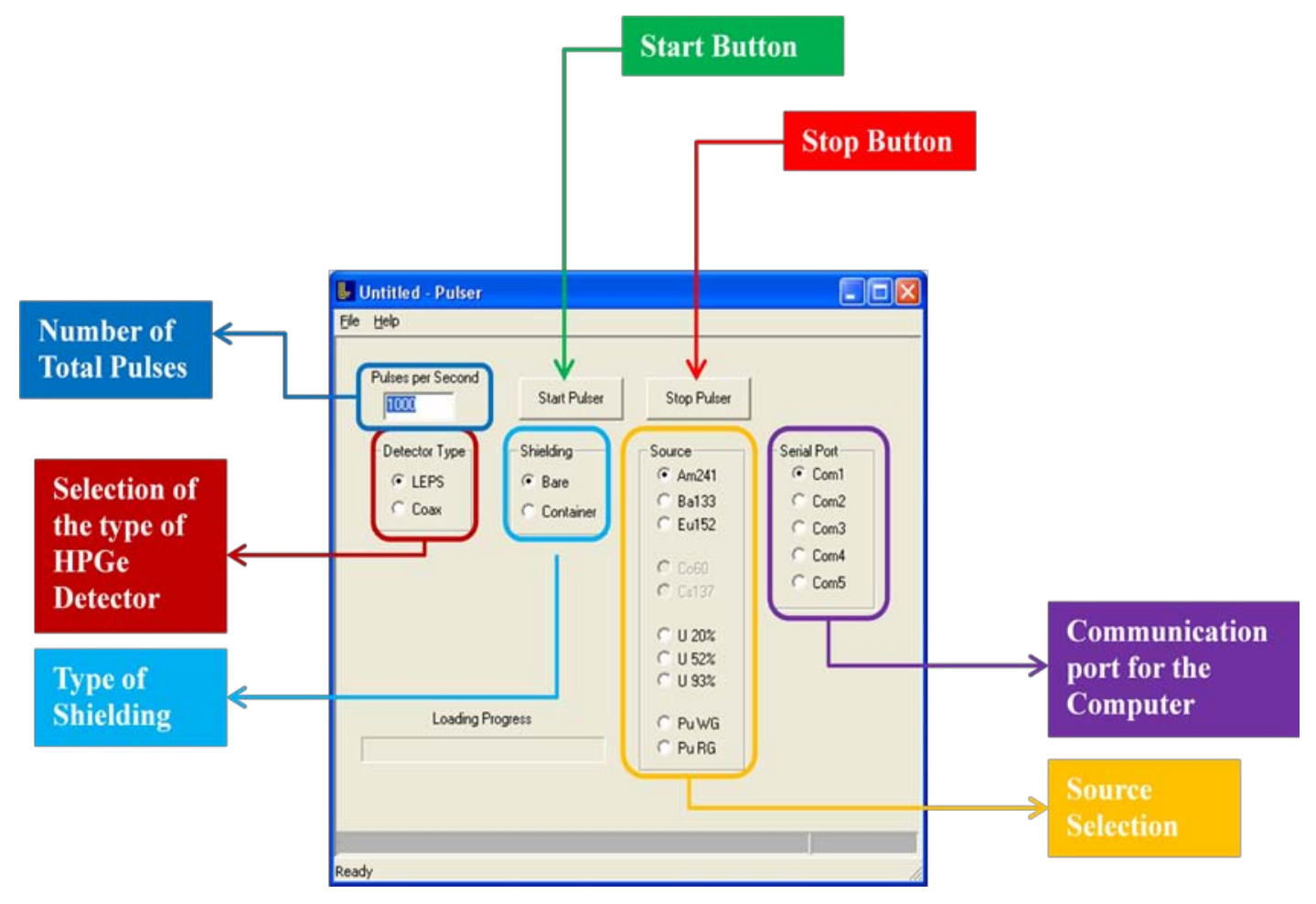

FIGURE 6: GRAPHICAL USER INTERFACE FOR THE SYNTHETIC GAMMA RAY SOURCE

\section{CONCLUSION}

This report presented a description of a Synthetic Gamma Ray Source. The synthetic source is simple to operate and can simulate a wide variety of sources.

\section{BIBLIOGRAPHY}

Breismeister, J. "MCNP- A General Monte Carlo N-Particle Transport Code." LA-12625-M, Los Alamos National Laboratory Report, Los Alamos, NM, 1991.

Gosnell, Thomas B. "Automated calculation of photon source emission from arbitrary mixtures of naturally radioactive heavy nuclides." Nuclear Instruments and Methods in Physics Research Section A 299, no. 1-3 (1990): 682-686.

Knoll, Glenn F. Radiation Detection and Measurement. New York: John Wiley \& Sons, 2000.

Mitchell, Dean J. Gamma Detector Response and Analysis Software. SAND92-0285-UC-702, Albuquerque, NM: Sandia National Laboratories, 1992.

Walker, Alastair J. "An Efficient Method for Generating Discrete Random Variables With General Distributions." ACM Transactions on Mathematical Software 3, no. 3 (1977): 253-256. 


\section{APPENDIX}

In order to follow this example the user will need the following: A PC with the Canberra Gamma Acquisition \& Analysis software loaded, a Canberra HPGe detector cable bundle, a Canberra portable MCA Inspector Model 1270 or equivalent, and a source of $110 \mathrm{~V}$ power. In addition the user will need a Windows PC with "Synthetic Gamma Ray Source.exe" installed in a folder with folder Energy that comes with this document installed in the same folder. The user is encouraged to make a shortcut of the executable and place that shortcut on the Desktop. It is suggested to that the user name the shortcut "Synthetic Gamma Ray Source" as shown in Figure 7.

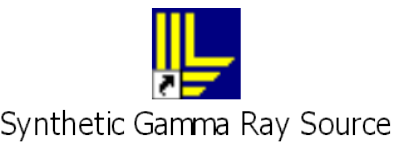

FIGURE 7: ICON THAT RUNS THE SYNTHETIC GAMMA RAY SOURCE

\section{Synthetic Gamma Ray Source SeT-UP}

- Connect $110 \mathrm{~V}$ line from the Synthetic Gamma Ray Source ${ }^{4}$ to power

- Attach a $50 \Omega$ cable to the coax connector on the backside of the Pulser

- Attach the other end of the $50 \Omega$ cable to the input of the MCA

- Attach a serial cable on the Pulser to the serial port on the PC that has the Synthetic Gamma Ray Source executable installed on it. ${ }^{5}$

\section{SETUP Using CANBerRa Inspector AND Genie GAMma ACQUiSITION \&}

\section{ANALYSIS SOFTWARE}

- Connect the "Energy" cable in the Canberra detector cable bundle to the "Preamp out" connector in the Pulser.

- Plug in the multi-pin connector to the back of the Inspector

- Connect the Inspector "Computer" PC output to the PC

- Connect power to the Inspector

- No other cable connections are required. Switch Inspector on.

- Power up the PC and start the Gamma Acquisition \& Analysis software

- Under the pull down menu under "File" select "Open Data Source", then in the "Type" field select "Detector", then select "DET01", and open.

- Verify MCA Acquire and Adjust settings agree with values in Appendix A. Adjust as needed under the pulldown menu under MCA or Display.

- Accept the rest of the default settings and select "OK”. The inspector is now ready to receive Pulser output.

\section{CALIBRATION}

\section{Calibration OF THE “COAX” Detector}

The first time the Synthetic Gamma Ray Source is used with an Inspector, just as with a simulated Coax HPGe detector, calibration must be performed on the MCA. The following denotes the calibration steps using Pulser output.

- Stop any ongoing data acquisition on the MCA/PC by selecting "Stop" and clear the screen

\footnotetext{
${ }^{4}$ Synthetic Gamma Ray Source will be described as "Pulser" in the Appendix.

${ }^{5}$ If the PC dos not have a serial port; a USB to serial adapter can be used.
} 
- In the Gamma Acquisition \& Analysis software on the PC set the Live Time to 300 sec

- On the Pulser: Select "Coax" as the Detector Type

- On the Pulser: Select "Cs137" as the Source

- $\quad$ On the Pulser: Select "Bare" for the Shielding

- On the Pulser input "10000" for Pulses per Second

- On the Pulser: Select Start Pulser

- In the Gamma Acquisition \& Analysis software on the PC: Select "Start". As the acquisition proceeds make sure that the 661.5 peak from ${ }^{137} \mathrm{Cs}$ is in about channel 2640; if it is not, increase or decrease the gain so that this is the case. This can be done in real-time with the cursor at 2640 to guide the eye. When the peak is in the proper channel clear the acquisition and let the MCA to run for the 300 sec live-time.

- On completion of the 300 sec data acquisition, DO NOT clear screen

- On the Pulser: Select Stop Pulser

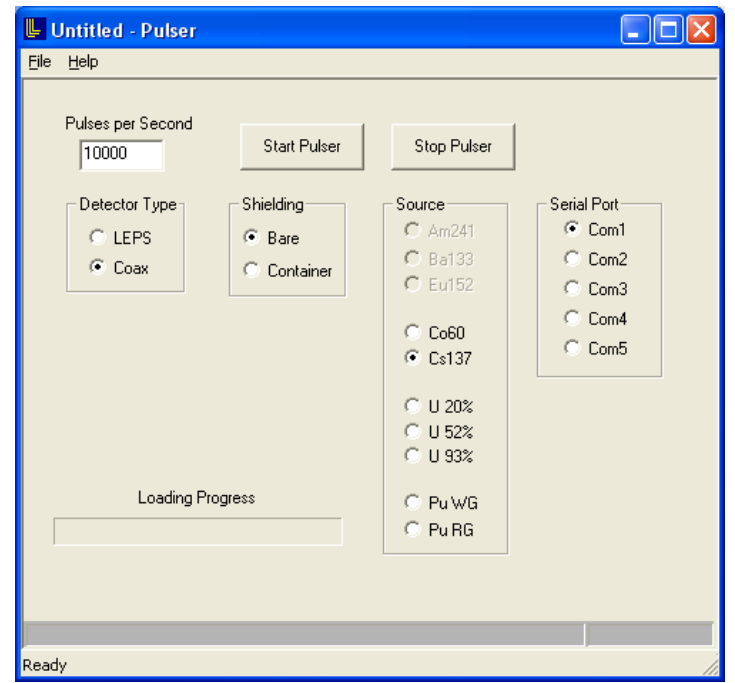

FIGURE 8: SCREEN SHOT FOR CS137 CALIBRATION

- In the Gamma Acquisition \& Analysis software: Select "Stop" but DO NOT clear screen

- In the Gamma Acquisition \& Analysis software on the PC set the Live Time to $600 \mathrm{sec}$

- On the Pulser: Select "Co60" as the Source

- On the Pulser input "10000" for Pulses per Second

- On the Pulser: Select Start Pulser 


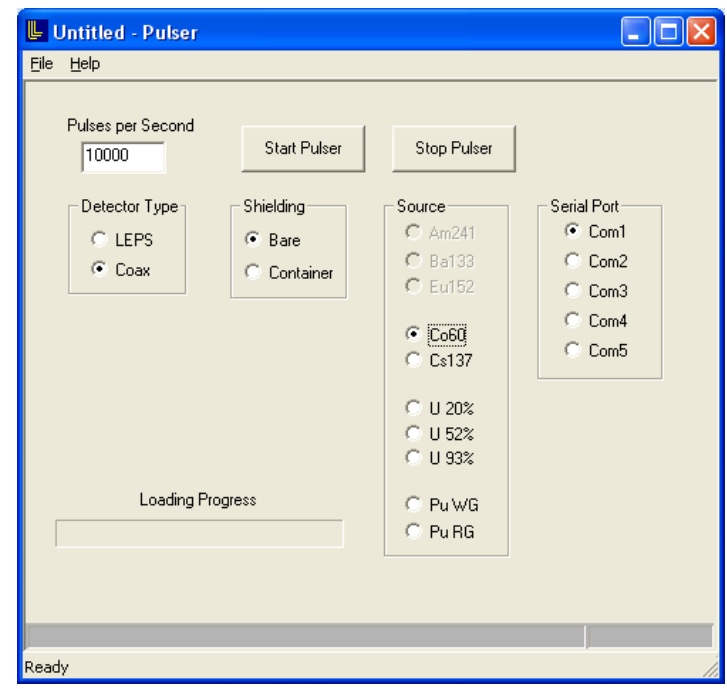

FIGURE 9: SCREEN SHOT FOR CO60 CALIBRATION

- On the Pulser and in the Gamma Acquisition \& Analysis software: Select "Start". Upon completion, the pulse height distributions for both ${ }^{137} \mathrm{Cs}$ and ${ }^{60} \mathrm{Co}$ are displayed on the PC.

- On the Pulser: Select Start Pulser

- When the acquisition is completed, In the Gamma Acquisition \& Analysis software: Select "Stop"

- On the Pulser: Select Start Pulser

- Using the Gamma Acquisition \& Analysis software: From the pull down menu select

- CalibratelEnergy Full $\backslash B y$ Nuclide list, and select Cs-137 and Co-60 from the nuclide list. Then select "Curser", then "OK".

- Place the curser at the peak for $661.5 \mathrm{keV}\left({ }^{137} \mathrm{Cs}\right.$ peak) and select "OK”

- Place the curser at the peak for $1073 \mathrm{keV}$ and do the same $\left({ }^{60} \mathrm{Co}\right.$ peak)

- $\quad$ Place the curser at the peak for $1132 \mathrm{keV}$ and do the same $\left({ }^{60} \mathrm{Co}\right.$ peak)

- $\quad$ Select "Save", which will store the energy calibrations in the Midfile of DET01.

- Continue with further analysis or exit the program. Spectra may be individually saved as CAM files in the GENIE software. When exiting the program, it is not necessary to save again if you have saved the calibration in the earlier step.

\section{CALIBRATION OF THE “LEPS” DETECTOR}

The first time the Synthetic Gamma Ray Source is used with an Inspector, just as with a LEPS HPGe detector, calibration must be performed on the MCA. The following denotes the calibration steps using Pulser output.

- Stop any ongoing data acquisition on the MCA/PC by selecting "Stop" and clear the screen

- In the Gamma Acquisition \& Analysis software on the PC set the Live Time to $300 \mathrm{sec}$

- On the Pulser: Select "LEPS" as the Detector Type

- On the Pulser: Select "Am241" as the Source

- On the Pulser: Select "Bare" for the Shielding

- On the Pulser input "10000" for Pulses per Second

- On the Pulser: Select Start Pulser

- In the Gamma Acquisition \& Analysis software on the PC: Select "Start". As the acquisition proceeds make sure that the 59.5 peak $^{6}$ from ${ }^{241} \mathrm{Am}$ is in about channel 1590. If it is not, increase or decrease the gain so

\footnotetext{
${ }^{6}$ There will be two gamma ray lines from the ${ }^{241} \mathrm{Am}$ source one at $26.3 \mathrm{keV}$ and one at $59.5 \mathrm{keV}$ the $26.3 \mathrm{kev}$ will be
} about an order of magnitude smaller than the $59.5 \mathrm{keV}$ peak. 
that this is the case. This can be done in real-time with the cursor at 1950 to guide the eye. When this is completed clear the acquisition. When the peak is in the proper channel, clear the acquisition and let the MCA to run for the 300 sec live-time.

- On completion of the 300 sec data acquisition, DO NOT clear screen

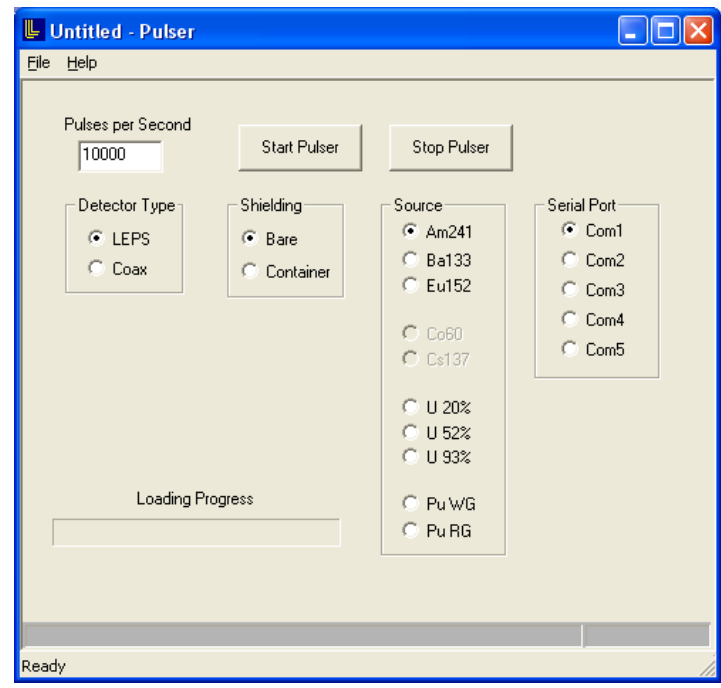

FIGURE 10: SCREEN SHOT FOR AM241 CALIBRATION

- In the Gamma Acquisition \& Analysis software: Select “Stop” but DO NOT clear screen

- In the Gamma Acquisition \& Analysis software on the PC set the Live Time to $600 \mathrm{sec}$

- On the Pulser: Select "152Eu" as the Source

- On the Pulser input "10000" for Pulses per Second

- On the Pulser: Select Start Pulser

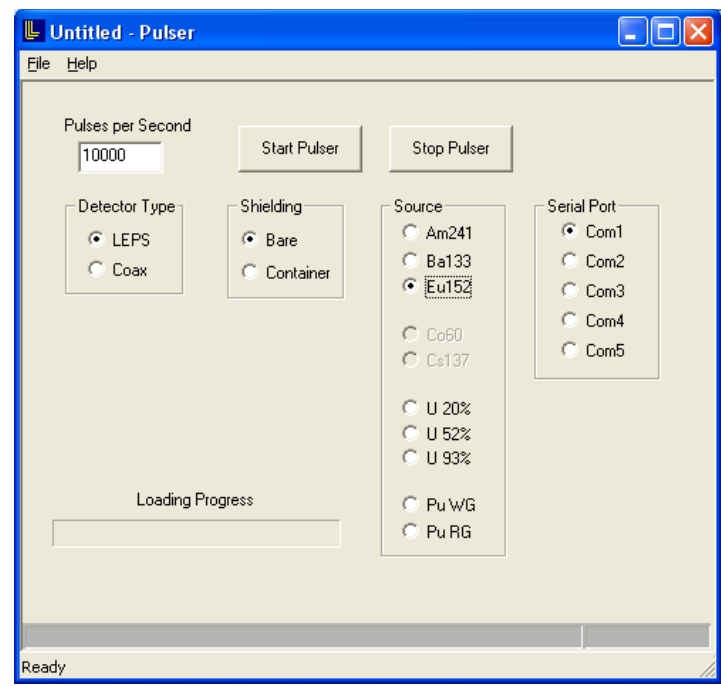

FIGURE 11: SCREEN SHOT FOR EU152 CALIBRATION 
- In the Gamma Acquisition \& Analysis software on the PC: Select "Start".

- Using the Gamma Acquisition \& Analysis software: From the pull down menu select

- Calibrate\Energy Full\By Nuclide list, and select Am-137 and Eu-152 from the nuclide list. Then select "Curser", then "OK".

- Place the curser at the peak for $59.5 \mathrm{keV}\left({ }^{241} \mathrm{Am}\right.$ peak) and select "OK"

- $\quad$ Place the curser at the peak for $121.8 \mathrm{keV}$ and do the same $\left({ }^{152} \mathrm{Eu}\right.$ peak)

- Select "Save”, which will store the energy calibrations in the Midfile of DET01.

- Continue with further analysis or exit the program. Spectra may be individually saved as CAM files in the GENIE software. When exiting the program, it is not necessary to save again if you have saved the calibration in the earlier step. 